\title{
ON CHEVALLEY-SHEPHARD-TODD'S THEOREM IN POSITIVE CHARACTERISTIC
}

\author{
ABRAHAM BROER \\ To Gerald Schwarz, on the occasion of his 60-th anniversary
}

\begin{abstract}
Let $G$ be a finite group acting linearly on the vector space $V$ over a field of arbitrary characteristic. The action is called coregular if the invariant ring is generated by algebraically independent homogeneous invariants and the direct summand property holds if there is a surjective $k[V]^{G}$-linear map $\pi: k[V] \rightarrow k[V]^{G}$.

The following Chevalley-Shephard-Todd type theorem is proved. Suppose $V$ is an irreducible $k G$-representation, then the action is coregular if and only if $G$ is generated by pseudo-reflections and the direct summand property holds.
\end{abstract}

\section{INTRODUCTION}

Let $V$ be a vector space of dimension $n$ over a field $k$. A linear transformation $\tau: V \rightarrow V$ is called a pseudo-reflection, if its fixed-points space $V^{\tau}=\{v \in V ; \tau(v)=v\}$ is a linear subspace of codimension one. Let $G<\mathrm{GL}(V)$ be a finite group acting linearly on $V$. Then $G$ acts by algebra automorphisms on the coordinate ring $k[V]$, which is by definition the symmetric algebra on the dual vector space $V^{*}$. We shall say that $G$ is a pseudo-reflection group if $G$ is generated by pseudo-reflections; it is called a non-modular group if $|G|$ is not divisible by the characteristic of the field. The action is called coregular if the invariant ring is generated by $n$ algebraically independent homogeneous invariants.

A well-known theorem of Chevalley-Shephard-Todd [2, Chapter 6] says that if the group is non-modular then $G$ is a pseudo-reflection group if and only if the action is coregular.

By a theorem of Serre [2, Theorem 6.2.2] the implication that coregularity of the action implies that $G$ is a pseudo-reflection group is true even without the condition of non-modularity. This is not true for the other implication: there are pseudo-reflection groups whose action is not coregular.

Coxeter, Shephard and Todd classified all pseudo-reflection groups in characteristic zero. More recently the irreducible pseudo-reflection groups were classified over any characteristic, by Kantor, Wagner, Zaleskiı̌ and Serežkin. Using this classification Kemper-Malle [6] decided which irreducible pseudo-reflection groups possess the coregular property and which do not. They observed that those irreducible pseudo-reflection groups that possess the coregularity

Date: October 30, 2018. 
property are exactly those such that the actions are coregular for all the point stabilizers of non-trivial subspaces.

We say that the direct summand property holds if there is a surjective $k[V]^{G}$-linear map $\pi: k[V] \rightarrow k[V]^{G}$ respecting the gradings. For a non-modular group the direct summand property always holds, because in that case we can take the transfer $\operatorname{Tr}^{G}$ as projection, defined by

$$
\operatorname{Tr}^{G}: k[V] \rightarrow k[V]^{G}: \operatorname{Tr}^{G}(f)=\sum_{\sigma \in G} \sigma(f) ;
$$

since for any invariant $f$ we have $\operatorname{Tr}^{G}\left(|G|^{-1} f\right)=f$. Also the coregular property implies the direct summand property.

In this article we show first that if the direct summand property holds for $G$ then the direct summand property holds for all the point-stabilizers of subspaces of $V$, cf. Theorem 1. Then using this and the results of Kemper-Malle we show for irreducible $G$-actions that the action is coregular if and only if $G$ is a pseudo-reflection group and the direct summand property holds, cf. Theorem 2, We conjectured before that this also holds without the irreduciblity condition, cf. [3]. Elsewhere we show that the converse is also true if $G$ is abelian, cf. [4].

In the first section we show that the direct summand property is inherited by pointstabilizers. In the second section we recall Kemper-Malle's classification of irreducible pseudo-reflection groups that are not coregular, and describe the other tools used in the proof of the main theorem. In the last section we give the details of the calculations.

\section{ThE DIRECT SUMMAND PROPERTY AND POINT STABILIZERS}

For elementary facts on the invariant theory of finite groups we refer to [2], for a discussion of the direct summand property and the different see [3]. The transfer map extends to the quotient field of $k[V]$. We recall that the (Dedekind) different $\theta_{G}$ of the $G$-action on $V$ can be defined as the largest degree homogeneous form $\theta_{G} \in k[V]$ such that $\operatorname{Tr}^{G}\left(\frac{f}{\theta_{G}}\right)$ is without denominator, i.e. $\operatorname{Tr}^{G}\left(\frac{f}{\theta_{G}}\right) \in k[V]^{G}$, for all $f \in k[V]$; it is unique up to a multiplicative scalar. The direct summand property holds if and only if there exists a $\tilde{\theta}_{G} \in k[V]$ such that $\operatorname{Tr}^{G}\left(\frac{\tilde{\theta}_{G}}{\theta_{G}}\right)=1$ and then we can take as $k[V]^{G}$-linear projection

$$
\pi: k[V] \rightarrow k[V]^{G}: \pi(f):=\operatorname{Tr}^{G}\left(\frac{\tilde{\theta}_{G} f}{\theta_{G}}\right) .
$$

In Kemper-Malle's classification Steinberg's classical result is often used saying that the coregular property is inherited by point stabilizers of linear subspaces. We shall prove that also the direct summand property is inherited by point stabilizers of linear subspaces.

The key point in the proof of both results is that the affine group $V^{G}$ acts on $V$ by translations, namely $\tau_{u}: v \mapsto v+u\left(u \in V^{G}, v \in V\right)$, commuting with the linear $G$-action.

Theorem 1. Let the finite group $G$ act linearly on the vector space $V$ over the field $k$ and let $H$ be the point-stabilizer of a linear subspace $U \subset V$. 
If the $G$ action on $V$ has the direct summand property then the $H$ action on $V$ also has the direct summand property.

Proof. We write $A:=k[V], C:=k[V]^{H}$ and $B=A^{G}$. The prime ideal generated by the linear forms vanishing on $U$ is denoted by $\mathfrak{P} \subset A$; its intersection with $C$ is the prime ideal $\mathfrak{q}=\mathfrak{P} \cap C$. The inertia subgroup of $\mathfrak{P}$ coincides with $H$ :

$$
H=\{\sigma \in G ;(\sigma-1)(A) \subseteq \mathfrak{P}\}
$$

Let $\theta_{G}$ and $\theta_{H}$ be the two (Dedekind) differents with respect to the $G$-action and the $H$-action on $V$. In particular $\operatorname{Tr}^{G}\left(\frac{A}{\theta_{G}}\right) \subseteq B$, and $\operatorname{Tr}^{H}\left(\frac{A}{\theta_{H}}\right) \subseteq C$.

Let $V^{\alpha} \subset V$ be a linear subspace of codimension one, defined as the zero set of the linear form $x_{\alpha}$. Then $x_{\alpha}$ divides $\theta_{G}$ if and only if there is a pseudo-reflection in $G$ with reflecting hyperplane $V^{\alpha}$, or in other words there exists a $g \in G$ such that for all $a \in A, g(a)-a \in x_{\alpha} A$. Now for such a pseudo-reflection $g$ we have

$$
g \in H \Longleftrightarrow V^{\alpha} \supseteq U \Longleftrightarrow x_{\alpha} \in \mathfrak{P}
$$

It follows that $\theta_{H}$ is the part of $\theta_{G}$ involving the powers of linear forms $x_{\alpha}$, such that $x_{\alpha} \in \mathfrak{P}$. Let $\theta_{G / H}$ be the part of $\theta_{G}$ involving the powers of linear forms $x_{\alpha}$, such that $x_{\alpha} \notin \mathfrak{P}$, then $\theta_{G}=\theta_{G / H} \cdot \theta_{H}$. So $\theta_{H}$ and $\theta_{G / H}$ are relatively prime, and more importantly $\theta_{G / H} \notin \mathfrak{P}$

The homogeneous element $\theta_{G}$ is a $G$-semi-invariant for some character $\chi: G \rightarrow k^{\times}$. Similarly $\theta_{H}$ is an $H$-semi-invariant. The quotient $\theta_{G / H}=\theta_{G} / \theta_{H}$ is an element of $A$, and is also an $H$-semi-invariant. So there is a power $\theta_{G / H}^{e}$ that is an absolute $H$-invariant, i.e., $\theta_{G / H}^{e} \in C$, but

$$
\theta_{G / H}^{e} \notin \mathfrak{P} \cap C=\mathfrak{q} .
$$

Assume now that $B$ is a direct summand of $A$ as graded $B$-module; hence there exists a homogeneneous $\tilde{\theta} \in A$ such that $\operatorname{Tr}^{G}\left(\frac{\tilde{\theta}}{\theta_{G}}\right)=1$. We have to prove that the action of $H$ also has the direct summand property, or that the ideal

$$
I_{H}:=\operatorname{Tr}^{H}\left(\frac{A}{\theta_{H}}\right) \subseteq C
$$

is in fact equal to $C$.

We shall first show that

$$
\theta_{G / H}^{e} \in I_{H} .
$$

Since $\theta_{G / H}^{e} \notin \mathfrak{q}$, it will follow that $I_{H} \not \subset \mathfrak{q}$. 
Let $g_{1}, \ldots, g_{s}$ be right coset representatives of $H$ in $G$, i.e., we have a disjoint union $G=\cup_{i=1}^{s} H g_{i}$. Then

$$
\begin{aligned}
\theta_{G / H}^{e} & =\theta_{G / H}^{e} \cdot \operatorname{Tr}^{G}\left(\frac{\tilde{\theta}}{\theta_{G}}\right) \\
& =\theta_{G / H}^{e} \cdot \operatorname{Tr}^{H}\left(\sum_{i=1}^{s} g_{i}\left(\frac{\tilde{\theta}}{\theta_{G}}\right)\right) \\
& =\operatorname{Tr}^{H}\left(\theta_{G / H}^{e} \cdot \sum_{i=1}^{s} g_{i}\left(\frac{\tilde{\theta}}{\theta_{G}}\right)\right) \\
& \left.=\operatorname{Tr}^{H}\left(\theta_{G / H}^{e} \cdot \frac{\sum_{i=1}^{s} \chi^{-1}\left(g_{i}\right) g_{i}(\tilde{\theta})}{\theta_{G}}\right)\right) \\
& \left.=\operatorname{Tr}^{H}\left(\frac{\theta_{G / H}^{e-1} \cdot \sum_{i=1}^{s} \chi^{-1}\left(g_{i}\right) g_{i}(\tilde{\theta})}{\theta_{H}}\right)\right) \in \operatorname{Tr}^{H}\left(\frac{A}{\theta_{H}}\right)=I_{H} .
\end{aligned}
$$

Suppose now that $I_{H}$ is a proper ideal. Since it is a homogeneous ideal of $C$ it is then contained in the maximal homogeneous ideal $\mathfrak{M}_{0}$ of $A$, the ideal of polynomials all vanishing at the origin $0 \in V$. We shall show that then even $I_{H} \subseteq \mathfrak{P}$, which is a contradiction.

To prove this we can assume that $k$ is algebraically closed. If $u \in U$, then the affine transformation $\tau_{u}: v \mapsto v+u$ commutes with the linear $H$ action, since

$$
\tau_{u}(h \cdot v)=h \cdot v+u=h \cdot(v+u)=h \cdot\left(\tau_{u}(v)\right) .
$$

So it induces an algebra automorphism $\alpha=\tau_{u}^{*}$ of $A$ commuting with the $H$-action, by

$$
\alpha(f)(v)=\left(\tau_{u}^{*} \cdot f\right)(v):=f(v-u)
$$

moving the maximal ideal $\mathfrak{M}_{0}$ into the maximal ideal $\mathfrak{M}_{u}$ of polynomials in $A$ vanishing at $u$. It also commutes with $\operatorname{Tr}^{H}$, and fixes the linear forms of $\mathfrak{P}$, so it fixes $\theta_{H}$. But then

$$
I_{H}=\operatorname{Tr}^{H}\left(\frac{A}{\theta_{H}}\right)=\operatorname{Tr}^{H}\left(\frac{\alpha(A)}{\theta_{H}}\right)=\alpha\left(\operatorname{Tr}^{H}\left(\frac{A}{\theta_{H}}\right)\right) \subseteq \alpha\left(\mathfrak{M}_{0}\right) \subseteq \mathfrak{M}_{u}
$$

So $I_{H} \subseteq \cap_{u \in U} \mathfrak{M}_{u}$. By Hilbert's Nullstellensatz $\mathfrak{P}=\cap_{u \in U} \mathfrak{M}_{u}$ and

$$
I_{H} \subseteq \mathfrak{P} \cap C=\mathfrak{q}
$$

This is a contradiction, so $I_{H}$ is not a proper ideal, i.e.,

$$
\operatorname{Tr}^{H}\left(\frac{A}{\theta_{H}}\right)=C,
$$

which implies that the direct summand property holds for the $H$ action. 


\section{MAIN RESUlT AND TOOLS FOR THE PROOF}

In this section we describe the tools we used to prove our main theorem.

Theorem 2. Let $G$ be an irreducible pseudo-reflection group group acting on $V$. Then the action is coregular if and only if $G$ is a pseudo-reflection group and the direct summand property holds.

It is already known that if the action is coregular then $G$ is a pseudo-reflection group and the direct summand property holds; it follows from Serre's theorem [2, Theorem 6.2.2]. For the other direction we use Kemper-Malle's classification of irreducible pseudo-reflection groups not having the coregular property. We shall use their notation.

Theorem 3 (Kemper-Malle [6]). Let $G$ be an irreducible pseudo-reflection group group. Then it does not have the coregular property if and only if it occurs in the following list.

(I) (Unitary pseudo-reflection groups) $\mathrm{SU}_{n}(q) \leq G \leq \mathrm{GU}_{n}(q), n \geq 4$, and $\mathrm{SU}_{3}(q) \leq G<$ $\mathrm{GU}_{3}(q)$.

(II) (Symplectic pseudo-reflection groups) $\operatorname{Sp}_{n}(q), n \geq 4$ and $n=2 m$ even.

(III-a) (Orthogonal reflection groups of odd characteristic) $q$ odd: $\Omega_{n}^{( \pm)}(q)<G \leq \mathrm{GO}_{n}^{( \pm)}(q)$, except $\mathrm{GO}_{3}(q), \mathrm{R}^{+} \mathrm{O}_{3}(q), \mathrm{GO}_{4}^{-}(q)$.

(III-b) (Orthogonal pseudo-reflection groups of even characteristic) q even: $\mathrm{SO}_{2 m}^{( \pm)}(q)$, $2 m \geq 4$, except $\mathrm{SO}_{4}^{-}(q)$.

(IV) (Symmetric groups) $\mathfrak{S}_{n+2}, p \mid(n+2), n \geq 3$.

(V) (Exceptional cases) (i) $W_{3}\left(G_{30}\right)=W_{3}\left(H_{4}\right)$, (ii) $W_{3}\left(G_{31}\right)$, (iii) $W_{5}\left(G_{32}\right)$, (iv) $W_{3}\left(G_{36}\right)=$ $W_{3}\left(E_{7}\right)$, (v) $W_{3}\left(G_{37}\right)=W_{3}\left(E_{8}\right)$, (vi) $W_{5}\left(G_{37}\right)=W_{5}\left(E_{8}\right)$ and (vii) $W_{2}\left(G_{34}\right)=3 \cdot U_{4}(3) \cdot 2_{2}$.

Remark. In comparing Kemper-Malle's calculations with ours the reader should be aware that they work with the symmetric algebra of $V$ and we with the coordinate ring of $V$. See also the comments in [5] on Kemper-Malle's article.

2.1. Tools. To prove our theorem we shall exhibit for every pseudo-reflection group in Kemper-Malle's list an explicit point-stabilizer $H$ such that for the $H$-action on $V$ the direct summand property does not hold. Then by Theorem 1 the $G$-action on $V$ does not have the direct summand property either.

In most cases we found a point stabilizer $H$ that is a $p$-group. Then we can use the following tools to show that the $H$-action does not have the direct summand property.

If $H$ is a $p$-group acting on $V$ and the direct summand property holds then $H$ is generated by its transvections, cf. [3, Corollary 4]. So if $H$ is not generated by transvections then the direct summand property does not hold.

Often $H$ is abelian. Then we can use that for abelian pseudo-reflection groups the direct summand property holds if and only if the action is coregular, cf. [4].

For induction purposes the following trivial remark is useful. Let $H$ be a group. We shall say that two $k H$-modules $V_{1}$ and $V_{2}$ are equivalent if one is obtained from the other by adding 
a trivial direct summand, for example $V_{2} \simeq V_{1} \oplus k^{r}$. Then the action on one is coregular (has the direct summand property, et cetera) if and only if the other is coregular (has the direct summand property, et cetera).

Sometimes the following is useful to disprove coregularity. If the action is coregular with fundamental degrees $d_{1}, \ldots, d_{n}$. Then $\prod_{i=1}^{n} d_{i}=|G|$ and $\sum_{i=1}^{n} d_{i}=\delta_{G}+n$, e.g. [3], §2.5], where $\delta_{G}$ is the differential degree, i.e., the degree of the different $\theta_{G}$. We give two examples.

Example 1. (i) Let $k=\mathbb{F}_{q^{2}}, q=p^{r}$ and $V=k^{2 n}, 2 n \geq 4$, with standard basis $e_{1}, \ldots, e_{2 n}$. Consider the group

$$
G_{n}:=\left\{\left(\begin{array}{cc}
I & O \\
B & I
\end{array}\right) ; \bar{B}=-B^{T}\right\},
$$

where $B$ is an anti-hermitian $n \times n$ matrix with coefficients in $\mathbb{F}_{q^{2}}$, and where $\bar{B}_{i j}:=B_{i j}^{q}$. It is normalized by

$$
N:=\left\{\left(\begin{array}{cc}
A & O \\
O & \bar{A}^{-T}
\end{array}\right) ; A \in \operatorname{GL}\left(n, \mathbb{F}_{q^{2}}\right)\right\}
$$

and $N$ acts transitively on the $\frac{q^{2 n}-1}{q^{2}-1}$ hyperplanes containing the subspace $<e_{n+1}, \ldots, e_{2 n}>$. Take the hyperplane $\left\langle e_{2}, \ldots, e_{2 n}>\right.$; its point-stabilizer $H$ consists of all matrices in $G_{n}$ where all coefficients of $B$ are 0 except possibly $b_{11}$. Its invariant ring has differential degree $q-1$. So the differential degree of the $G_{n}$ action is

$$
\delta_{G_{n}}=\frac{q^{2 n}-1}{q^{2}-1}(q-1)
$$

Let $K$ be the point stabilizer of the subspace $<e_{3}, \ldots, e_{2 n}>$. Then $K \simeq G_{2}$ and the action of $K$ on $V$ is equivalent to the $G_{2}$ action on $k^{4}$. Suppose the $G_{2}$-action is coregular with fundamental degrees $d_{1}, \ldots, d_{4}$. Then $d_{1}=d_{2}=1$, since the first two coordinate functions are invariants. And $d_{1} d_{2} d_{3} d_{4}=\left|G_{2}\right|=q^{4}$. So $\left(d_{1}, d_{2}, d_{3}, d_{4}\right)=\left(1,1, p^{r}, p^{s}\right)$ for some $r \geq 1, s \geq 1$ and $\sum_{i} d_{i}=\delta_{G_{2}}+n$, i.e. $1+1+p^{r}+p^{s}=q^{3}-q^{2}+q-1+4$. Implying that $2 \equiv 3$ modulo $p$, which is a contradiction. So the action of $K$ is not coregular. Since it is abelian it does not have the direct summand property either.

Conclusion: the action of transvection group $G_{n}$ on $k^{2 n}, n \geq 2$, is not coregular and does not have the direct summand property.

(ii) Let $k=\mathbb{F}_{q}, q=p^{r}, V=k^{2 n}, 2 n \geq 4$, with standard basis $e_{1}, \ldots, e_{2 n}$. Consider the group

$$
G_{n}:=\left\{\left(\begin{array}{cc}
I & O \\
B & I
\end{array}\right) ; B=B^{T}\right\},
$$

where $B$ is a symmetric $n \times n$ matrix with coefficients in $\mathbb{F}_{q}$. It is normalized by

$$
N:=\left\{\left(\begin{array}{cc}
A & O \\
O & A^{-T}
\end{array}\right) ; A \in \operatorname{GL}\left(n, \mathbb{F}_{q}\right)\right\} .
$$


As in (i) we can calculate the differential degree of the $G_{n}$-action, it is

$$
\delta_{G_{n}}=\frac{q^{n}-1}{q-1}(q-1)=q^{n}-1 .
$$

Then as in (i) we can consider the point-stablizer $H$ of $\left\langle e_{3}, \ldots, e_{2 n}\right\rangle$ and obtain the same conclusion. The action of $G_{n}$ on $k^{2 n}, n \geq 2$ is not coregular and does not have the direct summand property.

And the last tool we shall use to prove that the direct summand property does not hold is the following. If the direct summand property holds for the action of $G$ on $V$ and $J \subset k[V]^{G}$ an ideal, then $J=(J \cdot k[V]) \cap k[V]^{G}$, cf.[3, Proposition 6(ii)]. We give an example of its use.

Example 2. Let $k=\mathbb{F}_{q^{2}}$ and $V=k^{3}$ with standard basis $e_{1}, e_{2}, e_{3}$ and coordinate functions $x_{1}, x_{2}, x_{3}$. Let $H$ be the point stabilizer of $<e_{3}>$ inside $\mathrm{GU}_{3}(q)$, and $\tilde{H}$ the point stabilizer of $<e_{3}>$ inside $\mathrm{SU}_{3}(q)$. Or explicitly,

$$
H=\left\{\left(\begin{array}{lll}
1 & 0 & 0 \\
a & b & 0 \\
c & d & 1
\end{array}\right) ; b^{q+1}=1, d=-b a^{q}, c+c^{q}+a^{q+1}=0\right\}
$$

and $\tilde{H}$ is the normal subgroup where $b=1$. Let $\eta$ be a primitive $q+1$-st root of unity and

$$
\tau:=\left(\begin{array}{ccc}
1 & 0 & 0 \\
0 & \eta^{-1} & 0 \\
0 & 0 & 1
\end{array}\right)
$$

Then $H=<\tilde{H}, \tau>$.

Both point stabilizer groups have the algebraically independent invariants

$$
x_{1}, F:=x_{1} x_{3}^{q}+x_{2}^{q+1}+x_{3} x_{1}^{q} \text { and } N\left(x_{3}\right):=\prod_{\sigma \in H / \operatorname{Stab}_{H}\left(x_{3}\right)} \sigma\left(x_{3}\right),
$$

of degrees $1, q+1$ and $q^{3}$ respectively. Since $|H|=(q+1) q^{3}$ they form a generating set of the invariant ring $k[V]^{H}$, cf. [6, Proof of Proposition 3.1].

Let

$$
h:=N\left(x_{2}\right)=\prod_{\sigma \in \tilde{H} / \operatorname{Stab}_{\tilde{H}}\left(x_{2}\right)} \sigma\left(x_{2}\right)=x_{2}\left(x_{2}^{q^{2}-1}-x_{1}^{q^{2}-1}\right) .
$$

Then by construction $h$ is $\tilde{H}$-invariant and $\tau \cdot h=\eta h$. So $h^{q+1}$ is the smallest power of $h$ that is $H$-invariant. Let $f$ be any $\tilde{H}$-invariant such that $\tau \cdot f=\eta f$. Since $\tau$ is a pseudo-reflection we have that $\tau(f)-f=(\eta-1) f$ is divisible by $x_{2}$. Since $f$ is also $\tilde{H}$-invariant it is also divisible by every $\sigma\left(x_{2}\right), \sigma \in \tilde{H}$, so is divisible by $h$. Using powers $h^{i}$ we get similar results for other $H$-semi-invariants. We get

$$
k[V]^{\tilde{H}}=\oplus_{i=0}^{q} k[V]^{H} h^{i}
$$


and so

$$
k[V]^{\tilde{H}}=k\left[x_{1}, F, N\left(x_{3}\right), h\right] ;
$$

in particular it is a hypersurface ring. Similarly for $H_{m}=<\tilde{H}, \tau^{m}>$, for $m \mid(q+1)$, we get $k[V]^{H_{m}}=k\left[x_{1}, F, N\left(x_{3}\right), h^{(q+1) / m}\right]$. In any case

$$
\left(x_{1}, F, N\left(x_{3}\right), h^{(q+1) / m}\right) k[V]=\left(x_{1}, x_{2}^{q+1}, x_{3}^{q^{3}}\right) k[V]=\left(x_{1}, F, N\left(x_{3}\right)\right) k[V] .
$$

If the direct summand property holds for $H_{m}$ acting on $V$, then for any ideal $J \subset k[V]^{H_{m}}$ we have $J=(J \cdot k[V]) \cap k[V]^{H_{m}}$. In particular, it follows for the maximal homogeneous ideal $k[V]_{+}^{H_{m}}$ of $k[V]^{H_{m}}$ that

$$
k[V]_{+}^{H_{m}}=\left(x_{1}, F, N\left(x_{3}\right), h^{(q+1) / m}\right) k[V]^{H_{m}}=\left(x_{1}, F, N\left(x_{3}\right)\right) k[V]^{H_{m}} .
$$

So $x_{1}, F$ and $N\left(x_{3}\right)$ generate the maximal homogeneous ideal $k[V]_{+}^{H_{m}}$ and also the algebra $k[V]^{H_{m}}$. But this is a contradiction if $m \neq q+1$. Conclusion: the action of $H_{m}$ on $V=k^{3}$ does not satisfy the direct summand property if $m \mid(q+1)$ and $m \neq q+1$.

\section{Details}

In this last section we shall establish explicitly for every pseudo-reflection group not having the coregular property in Kemper-Malle's list in Theorem 3 a point stabilizer not having the direct summand property. For more information on some of the involved classical groups, for example Witt's theorem, see [1].

3.1. Families. (I) (Unitary pseudo-reflection groups) $\mathrm{SU}_{n}(q) \leq G \leq \mathrm{GU}_{n}(q), n \geq 4$, and $\mathrm{SU}_{3}(q) \leq G<\mathrm{GU}_{3}(q)$.

Let first $n=2 m \geq 4$ be even. Then there is a basis $e_{1}, \ldots, e_{2 m}$ of $V=\mathbb{F}_{q^{2}}^{n}$ such that the associated Gram matrix is

$$
J=\left(\begin{array}{ll}
O & I \\
I & O
\end{array}\right),
$$

where $I$ is the identity $m \times m$-matrix and $O$ the zero $m \times m$-matrix. So an $n \times n$ matrix $g$ with coefficients in $\mathbb{F}_{q^{2}}$ is in $\mathrm{GU}_{n}(q)$ if and only if $g^{T} J \bar{g}=J$, where $\bar{g}$ is the matrix obtained from $g$ by raising all its coefficients to the $q$-th power. Let $H$ be the point stabilizer in $G U_{n}(q)$ of the maximal isotropic subspace $U=<e_{m+1}, \ldots, e_{n}>$, so

$$
H=\left\{\left(\begin{array}{cc}
I & O \\
B & I
\end{array}\right) ; \bar{B}=-B^{T}\right\} .
$$

If $\mathrm{SU}_{n}(q) \leq G \leq \mathrm{GU}_{n}(q)$ then $H$ is also the point stabilizer in $G$ of $U$, since the index of $G$ in $\mathrm{GU}_{n}(q)$ is relatively prime to $p$ and $H$ is a $p$-group. We encountered this group in example 1(i), and we conclude that the direct summand property does not hold for $H$.

If $n=2 m+1 \geq 5$ is odd, then the stabilizer in $\mathrm{SU}_{n}(q) \leq G \leq \mathrm{GU}_{n}(q)$ of a non-singular vector is a reflection group $\mathrm{SU}_{2 m}(q) \leq G_{1} \leq \mathrm{GU}_{2 m}(q)$, so we can reduce to the even case, which we just handled. 
For $\mathrm{SU}_{3}(q) \leq G<\mathrm{GU}_{3}(q)$, see example 2. This is one of the rare cases where no pointstablizer could be found that was a $p$-group not having the direct summand property.

(II) (Symplectic pseudo-reflection groups) $\operatorname{Sp}_{n}(q), n \geq 4$ and $n=2 m$ even. There is a basis $e_{1}, \ldots, e_{2 m}$ of $\mathbb{F}_{q}^{n}$ such that the associated Gram-matrix is

$$
J=\left(\begin{array}{cc}
O & I \\
-I & O
\end{array}\right)
$$

where $I$ is the identity $m \times m$-matrix and $O$ the zero $m \times m$-matrix. So an $n \times n$ matrix $g$ with coefficients in $\mathbb{F}_{q}$ is in $\operatorname{Sp}_{n}(q)$ if and only if $g^{T} J g=J$. Let $H$ be the point stabilizer of the maximal isotropic subspace $U=<e_{m+1}, \ldots, e_{n}>$, so

$$
H=\left\{\left(\begin{array}{cc}
I & O \\
B & I
\end{array}\right) ; B=B^{T}\right\}
$$

We encountered this group in example 1(ii), and conclude that the direct summand property does not hold for $H$.

(III-a) (Orthogonal reflection groups of odd characteristic) $q$ odd: $\Omega_{n}^{( \pm)}(q)<G \leq \mathrm{GO}_{n}^{( \pm)}(q)$, except $\mathrm{GO}_{3}(q), \mathrm{R}^{+} \mathrm{O}_{3}(q), \mathrm{GO}_{4}^{-}(q)$.

Let $V=\mathbb{F}_{q}^{n}$. If $n=2 m$ is even, then $V$ admits two equivalence classes of non-degenerate quadratic forms distinguished by their sign; they are not similar. We get two orthogonal groups $\mathrm{GO}_{2 m}^{ \pm}(q)$. If $n=2 m+1$ is odd then there are also two equivalence classes of quadratic forms, but they are similar. For our purposes we need not distinguish the two (classes of) orthogonal groups, we write $\mathrm{GO}_{2 m+1}(q)$. In any case the orthogonal group does not contain transvections and contains two types of reflections (i.e. pseudo-reflections of order two). If $\sigma$ is a reflection, then its center $(\sigma-1)(V)$ is a one dimensional nonsingular subspace $\langle u\rangle$. Conversely, to any one dimensional nonsingular subspace $\langle u\rangle$ there corresponds a unique reflection. The orthogonal complement $\langle u\rangle^{\perp}$ is an irreducible orthogonal space and there are two possibilities, so by Witt's lemma there are exactly two conjugacy classes of nonsingular subspaces $\langle u\rangle$, hence two conjugacy classes of reflections. Each conjugacy class generates a normal reflection subgroup of the full orthogonal group of index 2 . These are the three reflection groups we consider.

Let us first consider $n=2 m \geq 4$ and the reflection subgroups $G<\mathrm{GO}_{2 m}^{+}(q)$. So there is a basis $e_{1}, \ldots, e_{2 m}$ of $V$ such that the associated Gram matrix is

$$
J=\left(\begin{array}{ll}
O & I \\
I & O
\end{array}\right)
$$

where $I$ is the identity $m \times m$-matrix and $O$ the zero $m \times m$-matrix. So an $n \times n$ matrix $g$ with coefficients in $\mathbb{F}_{q}$ is in $\mathrm{GO}_{n}^{+}(q)$ if and only if $g^{T} J g=J$. Let $H$ be the point stabilizer 
in $G O_{n}^{+}(q)$ of the maximal isotropic subspace $U=<e_{m+1}, \ldots, e_{n}>$, then

$$
H=\left\{\left(\begin{array}{cc}
I & O \\
B & I
\end{array}\right) ; B=-B^{T}\right\} .
$$

If $G$ is any of the reflection groups associated to $\mathrm{GO}_{n}^{+}(q)$ then its index is 1 or 2 , so $H$ is also the point stabilizer in $G$ of $U$. Since $H$ is a non-trivial $p$-group and does not contain pseudo-reflections it follows that the direct summand property does not hold for $H$.

If $n=2 m+1 \geq 5$ is odd, then there is a non-singular vector $u$ such that the point stabilizer of $\left\langle u>\right.$ in the reflection group $G<\mathrm{GO}_{2 m+1}(q)$ of index $\leq 2$ is a reflection group $G_{1}<\mathrm{GO}_{2 m}^{+}$of index $\leq 2$ acting irreducibly on $u^{\perp}$. We can use induction.

Consider now $n=2 m>4$ and the reflection subgroups $G<\mathrm{GO}_{2 m}^{-}(q)$ of index $\leq 2$. There are two linearly independent non-singular vectors $u_{1}, u_{2}$ such that the point stabilizer of $<u_{1}, u_{2}>$ in the reflection group $G<\mathrm{GO}_{2 m}^{-}(q)$ of index $\leq 2$ is a reflection group $G_{1}<\mathrm{GO}_{2 m-2}^{+}$of index $\leq 2$ acting irreducibly on $<u_{1}, u_{2}>^{\perp}$. We can reduce to the earlier case.

Consider $\mathrm{GO}_{3}(q)$, the orthogonal group with respect to the quadratic form $2 x_{1} x_{3}+x_{2}^{2}$. Let $H$ be the point-stabilizer of $\left\langle e_{3}>\right.$, then

$$
H=\left\{\left(\begin{array}{ccc}
1 & 0 & 0 \\
-b & a & 0 \\
\frac{-b^{2}}{2} & a b & 1
\end{array}\right) ; a^{2}=1, b \in \mathbb{F}_{q}\right\}
$$

The point-stabilizer $H^{-}$of $\mathrm{GO}_{3}^{-}(q)$ is the subgroup of $H$ where the coefficient $a=1$. So $H^{-}$ is a $p$-group without transvections, so the direct summand property does not hold for $\mathrm{H}^{-}$.

Let $H$ be the point stabilizer in $\mathrm{GO}_{4}^{-}(q)$ of an anisotropic line. Then $H$ is isomorphic to $\mathrm{GO}_{3}(q)$. So for at least one of the two reflection subgroups of $\mathrm{GO}_{4}^{-}(q)$ the point stabilizer $H^{\prime}$ of the anisotropic line is $\mathrm{GO}_{3}^{-}(q)$. So for that one the direct summand property does not hold. But both reflection subgroups of index two in $\mathrm{GO}_{4}^{-}(q)$ are conjugate inside the conformal orthogonal group; thus neither of them has the direct summand property.

(III-b) (Orthogonal pseudo-reflection groups of even characteristic) $q$ even: $\operatorname{SO}_{2 m}^{( \pm)}(q), 2 m \geq$ 4 , except $\mathrm{SO}_{4}^{-}(q)$. Let $V=\mathbb{F}_{q}^{n}$, where $n=2 m \geq 4$ is even. Then $V$ admits two equivalence classes of non-degenerate quadratic forms distinguished by their sign. We get two orthogonal groups $\mathrm{GO}_{2 m}^{ \pm}(q)$. Now the orthogonal groups are generated by transvections and do not contain reflections.

First consider $n=2 m \geq 4$ and a quadratic form with maximal Witt index. Then there is a basis $e_{1}, \ldots, e_{n}$ with dual basis $x_{1}, \ldots, x_{n}$ such that the quadratic form becomes $Q=$ $\sum_{i=1}^{m} x_{i} x_{m+i}$ and the Gram matrix is

$$
J=\left(\begin{array}{ll}
O & I \\
I & O
\end{array}\right)
$$


where $I$ is the identity $m \times m$-matrix and $O$ the zero $m \times m$-matrix. So an $n \times n$ matrix $g$ with coefficients in $\mathbb{F}_{q}$ is in $\mathrm{GO}_{n}^{+}(q)$ if and only if $Q=Q \circ g$ (and so $g^{T} J g=J$ ). Let $H$ be the point stabilizer in $G O_{n}^{+}(q)$ of the maximal isotropic subspace $U=<e_{m+1}, \ldots, e_{n}>$, so $H$ is the collection of matrices $\left(\begin{array}{cc}I & O \\ B & I\end{array}\right)$ such that $B_{i j}=B_{j i}$ if $1 \leq i \neq j \leq m$ and $B_{i i}=0$, for $1 \leq i \leq m$. Since $H$ is a $p$-group without pseudo-reflections, the direct summand property does not hold for $H$.

Next consider $n=2 m \geq 6$ and a quadratic form with non-maximal Witt index. Then there are two non-singular vectors $u_{1}, u_{2}$ such that the point stabilizer in $\mathrm{GO}_{n}^{-}(q)$ of $<u_{1}, u_{2}>$ is $\mathrm{GO}_{n-2}^{+}(q)$ acting irreducibly on $<u_{1}, u_{2}>^{\perp}$. And we can reduce to that case.

(IV) (Symmetric groups) $\mathfrak{S}_{n+2}, p \mid(n+2), n \geq 3$. Let $W=k^{m}$ be a vector space over a field of characteristic $p>0$ with basis $e_{1}, \ldots, e_{m}$; we assume $m \geq 5$. The symmetric group $\mathfrak{S}_{m}$ acts on $W$ by permuting the basis elements. The submodule of codimension one

$$
\tilde{V}=<e_{i}-e_{j} ; 1 \leq i<j \leq m>
$$

contains the submodule spanned by $v=\sum_{i=1}^{m} e_{i}$ if and only if $p$ divides $m$. We assume this; so $m=p m^{\prime}$ for some integer $m^{\prime}$ and we define $V$ to be the quotient module $\tilde{V} /<v>$ with dimension $n:=m-2 \geq 3$.

For $1 \leq j \leq m^{\prime}$ define $v_{j}:=\sum_{i=1}^{p} e_{p(j-1)+i}$, then $v=\sum_{j=1}^{m^{\prime}} v_{j}$ and each $v_{j} \in \tilde{V}$. Write $\tilde{U}_{1}=<v_{1}, \ldots, v_{m^{\prime}}>\subset \tilde{V}$ with image $U_{1}$ in $V$.

We remark that if for $\sigma \in \mathfrak{S}_{m}$ and $i$ it holds that $\sigma\left(v_{i}\right) \neq v_{i}$, then since $m \geq 5$ we have $\sigma\left(v_{i}\right)-v_{i} \notin\left\langle v>\right.$. So the point stabilizer of $U_{1}$ is the natural subgroup

$$
H:=\mathfrak{S}_{\{1,2, \ldots, p\}} \times \mathfrak{S}_{\{p+1, p+2, \ldots, 2 p\}} \times \ldots \times \mathfrak{S}_{\left\{\left(m^{\prime}-1\right) p+1, \ldots, m^{\prime} p\right\}} \simeq \mathfrak{S}_{p} \times \mathfrak{S}_{p} \times \ldots \times \mathfrak{S}_{p} .
$$

Suppose $p$ odd or $p=2$ and $m^{\prime}$ is even. Then $w:=\sum_{i=1}^{m} i e_{i} \in \tilde{V}$ and we define $\tilde{U}=$ $\tilde{U}_{1}+\left\langle w>\right.$ with image $U \subset V$. Let $\pi \in H$ such that $\pi(w)=w$ so if $\pi\left(e_{i}\right)=e_{j}$ then $i$ and $j$ are congruent modulo $p$, but this is only possible for $\pi \in H$ if $\pi$ is trivial. And if $\pi(w)-w \in\langle v\rangle$, or equivalently if there is a $c \in k$ such that

$$
\pi(w)-w=\sum_{i=1}^{m} i e_{\pi(i)}-\sum_{i=1}^{m} i e_{i}=\sum_{i=1}^{m}\left(\pi^{-1}(i)-i\right) e_{i}=c \sum_{i=1}^{m} e_{i}
$$

so $\pi^{-1}(i)=i+c$ for all $i$. So $c \in \mathbb{F}_{p}$ and $\pi$ is a power of

$$
\sigma:=(1,2,3, \ldots, p)(p+1, p+2, \ldots, 2 p) \cdots\left(\left(m^{\prime}-1\right) p+1,\left(m^{\prime}-1\right) p+2, \ldots, m\right) .
$$

We conclude that point stabilizer in $G$ of $\tilde{U}$ is now trivial, but the point stabilizer in $G$ of $U$ is not, it is generated by $\sigma$. On the other hand, the fixed point space of $\sigma$ is $U$. Since the dimension of $U$ is $m^{\prime}$, its codimension is

$$
m-2-m^{\prime}=(p-1) m^{\prime}-2>1
$$


(if $p=3$ then $m^{\prime} \geq 2$ and if $p=2$ then $m^{\prime} \geq 4$, by our assumptions), so $\sigma$ is not a pseudo-reflection. So we found a linear subspace whose point stabilizer is a cyclic $p$-group not containing a pseudo-reflection. So the direct summand property does not hold.

Let now $p=2$ and $m^{\prime}$ odd and we can assume $k=\mathbb{F}_{2}$. The point stabilizer $H$ is now an elementary abelian 2-group of order $2^{m^{\prime}}$ generated by the $m^{\prime}$ transpositions $(1,2),(3,4), \ldots,(m-$ $1, m)$. These are all transvections and the only pseudo-reflections contained in $H$. We shall show that its invariant ring is not polynomial. Take as basis $f_{1}, \ldots, f_{m-2}$ the images in $V$ of the vectors $e_{1}+e_{2}, e_{2}+e_{3}, \ldots, e_{m-2}+e_{m-1}$. Let $y_{1}, \ldots, y_{m}$ be the dual basis. Then the fixed point set $V^{H}$ is spanned by $f_{1}, f_{3}, f_{5}, \ldots, f_{m-3}$ and the fixed-point set $\left(V^{*}\right)^{H}$ by $y_{2}, y_{4}, \ldots, y_{m-2}$. Suppose $k[V]^{H}$ is a polynomial ring, and its fundamental degrees $d_{1}, d_{2}, \ldots, d_{m-2}$. We must have $|H|=2^{m^{\prime}}=d_{1} d_{2} \ldots d_{m-2}$ and the number of reflections must be $d_{1}+d_{2}+\ldots+d_{m^{\prime}-2}-\left(m^{\prime}-2\right)$. Since we have exactly $m^{\prime}-1$ independent linear invariants the fundamental invariants degrees there must be $m^{\prime}-2$ quadratic generating invariants and one of degree 4. So the number of reflections is $m^{\prime}-2+3=m^{\prime}+1$. But we have only $m^{\prime}$ reflections: a contradiction. So we found a linear subspace whose point stabilizer is an abelian $p$-group, whose ring of invariants is not a polynomial ring. Therefore the direct summand property does not hold either.

3.2. Exceptional cases. Kemper-Malle [6] made some explicit calculations to show that several exceptional irreducible reflection groups have a linear subspace whose point stabilizer is not generated by pseudo-reflections or at least its invariant ring is not polynomial. Using MAGMA we checked all these calculations and obtained the more precise result that all exceptional irreducible reflection groups without polynomial ring of invariants have in fact a linear subspace whose point stabilizer is an abelian p-group with an invariant ring that is not a polynomial ring, and so the direct summand property does not hold. In fact in most cases the point stabilizer is not even generated by pseudo-reflections.

(i) $W_{3}\left(G_{30}\right)=W_{3}\left(H_{4}\right)$. According to [6, p. 76] there is a point stabilizer of a twodimensional linear subspace that is cyclic of order 3. Since it was already known that the full pseudo-reflection group has no transvections, it follows that the point stabilizer is not generated by pseudo-reflections. Indeed, we checked that there is a two dimensional linear subspace with point stabilizer of order three and whose generator has two Jordan blocks of size 2 , hence this point stabilizer is an abelian $p$-group not generated by pseudo-reflections.

(ii) $W_{3}\left(G_{31}\right)$. According to [6, p. 76] there is a point stabilizer of a linear subspace that is not generated by pseudo-reflections and of order 48, which is not enough for our purposes. We checked that there is a unique orbit of length 960; fix a point $v$ in this orbit and let $H$ be its stabilizer (it is indeed of order 48). Now $H$ has 18 orbits of length 16 . We took one of them and took the stabilizer, say $K=H_{w}$. Then it turned out that $K$ has order 3 , generated by a $4 \times 4$ matrix whose Jordan form has two blocks of size 2 , so $K$ is the point stabilizer of $\langle v, w\rangle$ and is a $p$-group not generated by pseudo-reflections. 
(iii) $W_{5}\left(G_{32}\right)$. According to [6, p. 79] there is a one-dimensional linear subspace with point stabilizer a cyclic group of order 5. Since the full pseudo-reflection group was known to have no transvections it followed that this point stabilizer is not generated by pseudoreflections. Indeed we checked that there is a unique orbit whose stabilizers have order 5 and are generated by a $4 \times 4$ matrix whose Jordan form has one block of size 4 . So these stabilizers are not generated by pseudo-reflections, not even by 2-reflections. So by Kemper's theorem [5, Theorem 3.4.6], the invariant ring is not even Cohen-Macaulay.

(iv) $W_{3}\left(G_{36}\right)=W_{3}\left(E_{7}\right)$. According to [6, p. 78] there is a linear subspace whose point stabilizer of order 24 is not generated by pseudo-reflections. There is a unique orbit of length 672 ; let $N$ be the stabilizer of one of its points, say $v_{1}$. Now this group $N$ has several orbits of length 180, but only one of them has stablizers not generated by pseudo-reflections. Take $v_{2}$ in that orbit and take its stabilizer $N_{1}=N_{V_{1}}$ (so its order is 24 and is not generated by pseudo-reflections). Now this group $N_{1}$ has orbits whose stabilizers have order 3 . We took $v_{3}$ in one of those orbits, and took its stabilizer $N_{2} ; N_{2}$ was cyclic of order 3 , whose Jordan form has two blocks of size 2 and one of size three, so $N_{2}$ is the point stabilizer of $\left\langle v_{1}, v_{2}, v_{3}>\right.$ and its generator is not a pseudo-reflection, not even a 2-reflection. So the invariant ring is not even Cohen-Macaulay, [5, Theorem 3.4.6].

(v) $W_{3}\left(G_{37}\right)=W_{3}\left(E_{8}\right)$. According to [6, p. 78] there is a linear subspace having $W_{3}\left(E_{7}\right)$ as point stabilizer, so by the previous case it also has a linear subspace whose point stabilizer is a cyclic group of order 3, whose Jordan form has two blocks of size 2 and one each of size one and three. So the invariant ring is not even Cohen-Macaulay, [5, Theorem 3.4.6].

(vi) $W_{5}\left(G_{37}\right)=W_{5}\left(E_{8}\right)$. According to [6, p. 78] there is linear subspace whose point stabilizer is cyclic of order 5 . Since it was already known that the pseudo-reflection group does not contain any transvections it follows that this point stabilizer is not generated by pseudo-reflections. There is a unique orbit whose stabilizers have order 14400 , let $v_{1}$ be one of its points and $H$ its stabilizer. Now $H$ has a unique orbit with stabilizers of order 5 , let $v_{2}$ be one of its points and $N$ its stabilizer. Then $N$ is indeed cyclic of order 5 and the Jordan form of its generator has two blocks of size 4 , so $N$ is the point stabilizer of $\left\langle v_{1}, v_{2}\right\rangle$. So the invariant ring is not even Cohen-Macaulay, [5, Theorem 3.4.6]. Larger linear subspaces have a point stabilizer with polynomial ring of invariants.

(vii) $W_{2}\left(G_{34}\right)=3 \cdot U_{4}(3) \cdot 2_{2}$ (it has half the order of $G_{34}$ ). According to [6, p. 80] there is an explicit three dimensional linear subspace whose point stabilizer $K$ is a 2-group of order 32. The point stabilizer is abelian and generated by transvections, but we checked using MAGMA that the $K$-invariant rings of both $V$ and $V^{*}$ are non-polynomial (compare [5, p.107]).

As shown in [6, p.73] the remaining exceptional cases are all isomorphic as reflection groups to members of one of the families we already considered above. This finishes the proof of our main theorem. 


\section{REFERENCES}

[1] M. Aschbacher, Finite group theory, Second edition, Cambridge Studies in Advanced Mathematics, 10. Cambridge University Press, Cambridge, 2000.

[2] D.J. Benson, Polynomial invariants of finite groups, London Mathematical Society Lecture Note Series, 190. Cambridge University Press, Cambridge, 1993.

[3] A. Broer, The Direct Summand Property in Modular Invariant Theory, Transformation Groups 10 (2005) 5-27.

[4] A. Broer, Invariant theory of abelian transvection groups, submitted, preprint in arXiv:0709.0712 (math.AC).

[5] H. Derksen and G. Kemper, Computational invariant theory, Invariant Theory and Algebraic Transformation Groups, I. Encyclopaedia of Mathematical Sciences, 130. Springer-Verlag, Berlin, 2002

[6] G. Kemper and G. Malle, The finite irreducible linear groups with polynomial ring of invariants, Transformation Groups 2 (1997) 57-89.

[7] H. Nakajima, Invariants of finite groups generated by pseudo-reflections in positive characteristic, Tsukuba J. Math. 3 (1979), 109-122.

Département de mathématiques, et de statistique, Université de Montréal, C.P. 6128, succursale Centre-ville, Montréal (Québec), Canada H3C 3J7

E-mail address: broera@DMS.UMontreal.CA 